\title{
Rede de Antenas E Circularmente Polarizada
}

\author{
Laila F. Marzall, Delx C. Lunardi e J. C. da S. Lacava
}

\begin{abstract}
Resumo - Nestre trabalho é apresentado o projeto completo de uma rede de antenas circularmente polarizada, com faixa de passagem superior a $10 \%$. Tal projeto utiliza antenas $E$ como elementos irradiadores e divisores $\boldsymbol{T}$ e acoplador híbrido como componentes de seu sistema de alimentação.
\end{abstract}

Palavras-Chave - Antenas de microfita, polarização circular, redes de antena, sistema de alimentação.

Abstract - A complete design of a $10 \%$ bandwidth circularly polarized antenna array is performed. The design is based upon $E$-shaped printed antennas, with a quadrature hybrid and $T$ junctions forming the feed network.

Index Terms - Microstrip antennas, circular polarization, antenna arrays, beamforming network.

\section{INTRODUÇÃO}

A crescente demanda de serviços de radiodifusão e acesso a internet via satélite, que operam com larguras de banda cada vez maiores [1], vem exigindo o desenvolvimento de antenas circularmente polarizadas (CP) com larguras de banda equivalentes a destes sistemas. Este cenário parece não ser propício ao emprego de antenas de microfita, pois estas são irradiadores essencialmente faixa-estreita, seja do ponto de vista da variação de sua impedância de entrada com a freqüência, seja da variação de sua razão axial (RA) [2]. Entretanto, uma técnica capaz de melhorar o desempenho da largura de banda da RA de sistemas irradiantes é a conhecida alimentação seqüencial de elementos posicionados adequadamente em rede [2]-[4]. Na aplicação desta técnica o elemento irradiador e a topologia do sistema de alimentação da rede são dois fatores preponderantes de projeto, pois sabe-se que o desempenho da RA da referida rede é fortemente depende da largura de banda dos elementos que a compõem [5]. Neste contexto, este trabalho reporta o projeto completo de uma rede constituída por quatro antenas $E$ [6], com ênfase no seu circuito de alimentação.

O desenvolvimento do trabalho compreende quatro seções: na seção II são apresentadas as principais características de uma antena $E$ projetada para operar em $1,575 \mathrm{GHz}$. Esta freqüência foi escolhida por ser adequada às condições atuais de medida $\mathrm{e}$ de construção de protótipos do LAP (Laboratório de Antenas e Propagação do ITA). Na seção III propõe-se a topologia da rede seqüencial alimentada por fontes de corrente. $\mathrm{O}$ projeto do circuito de alimentação responsável por impor as correntes nas entradas das antenas é descrito na seção IV. Já na seção V o projeto completo é simulado, para que o impacto do circuito de alimentação sobre as características de irradiação da rede seja avaliado.

L.F. Marzall, J.C.S. Lacava, Laboratório de Antenas e Propagação - LAP, Instituto Tecnológico de Aeronáutica, São José dos Campos, SP, e-mail: laila@tsm.com.br, lacava@ita.br. D.C. Lunardi, Mectron, São José dos Campos, SP, e-mail: delxlunardi@yahoo.com.br. Este trabalho foi patrocinado pela CAPES e pela FINEP - Proieto Pseudo-Satélite.

\section{CARACTERÍSTICAS DA ANTENA $E$}

A geometria típica da antena $E$ [6] é mostrada na Fig. 1. Este irradiador foi o escolhido por possuir uma largura de banda de impedância maior do que as produzidas por geometrias simples, visto que possui duas freqüências de ressonância. Uma delas, a mais alta, é sintonizada pelo braço central. A outra, a mais baixa, é sintonizada pelos braços externos [7].

Como mencionado anteriormente, a rede em consideração deverá operar em 1,575 GHz. Neste item, portanto, são apresentados os resultados simulados de uma antena $E$ projetada no Ansoft Designer [8] para operar nesta mesma frequiência.

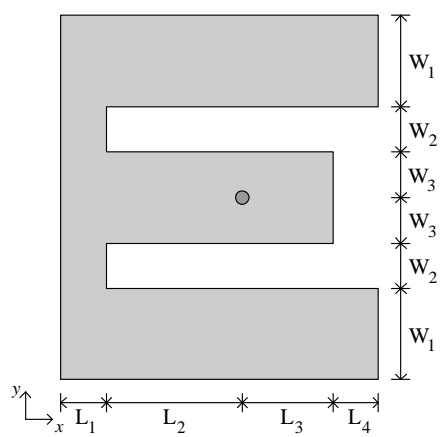

Fig. 1. Geometria típica da antena $E$.

Para obter um irradiador com adequada largura de banda, uma camada de $14,75 \mathrm{~mm}$ de ar é introduzida entre o plano de terra e o dielétrico de $0,6096 \mathrm{~mm}$ de espessura, $\varepsilon_{\mathrm{r}}=3,5$ e 0,0026 de tangente de perdas, que sustenta uma antena $E$ de dimensões $\mathrm{L}_{1}=13,2 \mathrm{~mm}, \mathrm{~L}_{2}=15,52 \mathrm{~mm}, \mathrm{~L}_{3}=24,12 \mathrm{~mm}, \mathrm{~L}_{4}=21,57 \mathrm{~mm}$, $\mathrm{W}_{1}=20,74 \mathrm{~mm}, \mathrm{~W}_{2}=10,21 \mathrm{~mm}$ e $\mathrm{W}_{3}=6,05 \mathrm{~mm}$. A referida antena é alimentada por uma via metálica de $1 \mathrm{~mm}$ de diâmetro. Na Fig. 2 é mostrada a curva obtida para a sua perda de retorno, da qual se nota que a antena possui duas ressonâncias, uma em $1,495 \mathrm{GHz}$ e outra em $1,656 \mathrm{GHz}$.

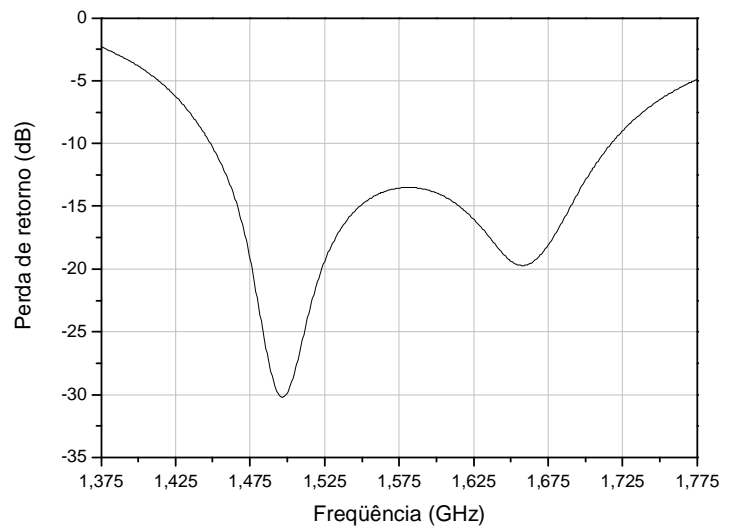

Fig. 2. Perda de retorno da antena $E$ projetada para $1,575 \mathrm{GHz}$. 
A largura de banda de impedância para pontos com perda de retorno menor ou igual a $-10 \mathrm{~dB}$ é de $16,9 \%$.

A antena $E$ possui uma assimetria geométrica que se reflete em seu diagrama de irradiação. Na Fig. 3 são apresentados os diagramas para as polarizações principal e cruzada, traçados nos planos xz e yz, de onde se verifica que a componente da polarização principal, no plano xz, sofre uma deformação e se torna de mesmo nível da componente da polarização principal, no plano yz, para valores de $\theta$ compreendidos entre $0^{\circ}$ e $60^{\circ}$. O ganho da antena $E$, na freqüência central, é da ordem de $8,8 \mathrm{~dB}$.

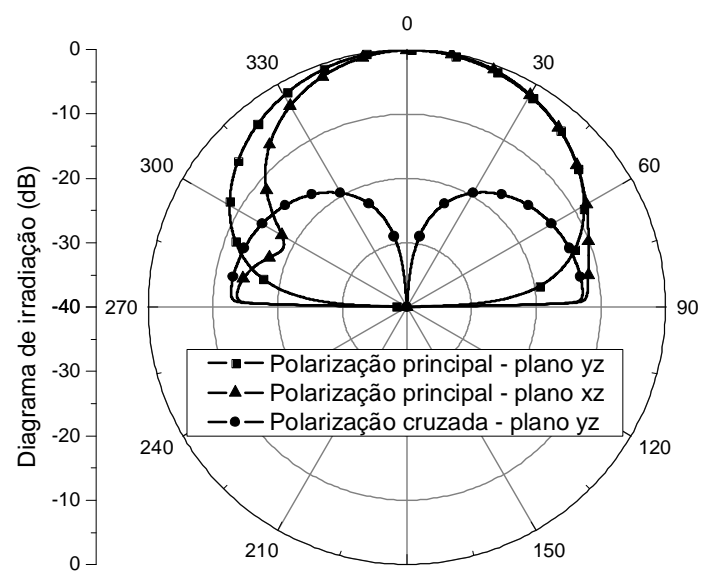

Fig. 3. Diagramas de irradiação da antena $E$ traçados nos planos principais.

\section{REDE CiRCUlarmente PolarizadA}

De acordo com [4], ondas eletromagnéticas CP podem ser obtidas empregando elementos linearmente polarizados em rede com alimentação seqüencial em rotação de fase. Nesta seção, este conceito é aplicado a uma rede constituída por antenas $E$, cuja disposição dos elementos é mostrada na Fig. 4. As dimensões destes elementos são as mesmas descritas no item anterior. Para gerar uma onda CP para a direita, com máximo na direção broadside, os elementos que compõem a rede devem ser alimentados com as correntes $I_{N}$ mostradas na Tabela I [7], $\operatorname{com} N=1,2,3$ ou 4.

A rede é, então, analisada na condição em que todos os elementos são alimentados por fontes de corrente. Na Fig. 5 são ilustrados os gráficos do módulo do coeficiente de reflexão, em função da freqüência, de cada uma dos elementos da rede. Nota-se que as curvas são equivalentes à do elemento isolado (Fig. 2), resultando numa largura de banda de $17 \%$ para perda de retorno de $-10 \mathrm{~dB}$.

TABELA I

CORRENTES DE ALIMENTAÇÃO DOS ELEMENTOS DA REDE.

\begin{tabular}{|c|c|}
\hline Elemento & $I_{N}(\mathrm{~A})$ \\
\hline 1 & $1 \angle 0^{\circ}$ \\
\hline 2 & $1 \angle 90^{\circ}$ \\
\hline 3 & $1 \angle 180^{\circ}$ \\
\hline 4 & $1 \angle 270^{\circ}$ \\
\hline
\end{tabular}

O comportamento das partes reais das impedâncias própria e mútua do primeiro elemento é mostrado na Fig. 6. Verificase uma alta isolação entre os elementos da rede, de acordo com [4]. Comportamento similar é observado para os outros elementos da rede.

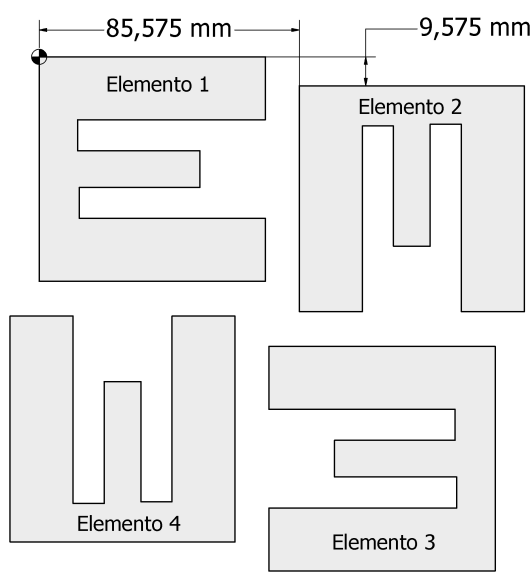

Fig. 4. Disposição das antenas $E$ em rede para gerar irradiação $\mathrm{CP}$.

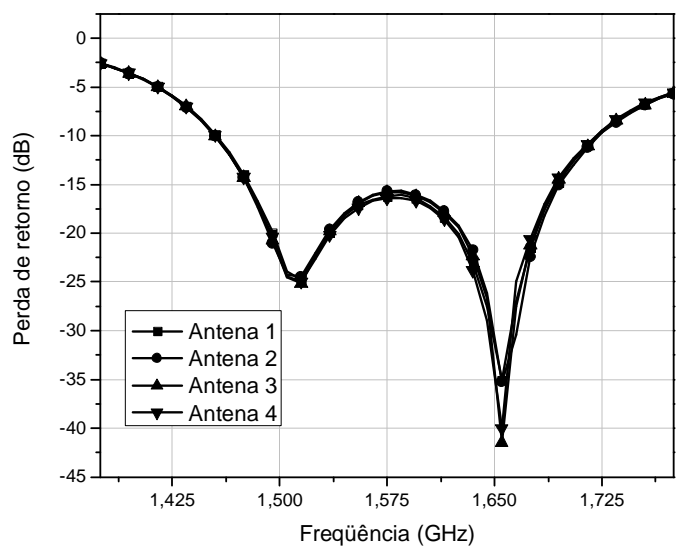

Fig. 5. Perdas de retorno das quatro antenas $E$ em rede.

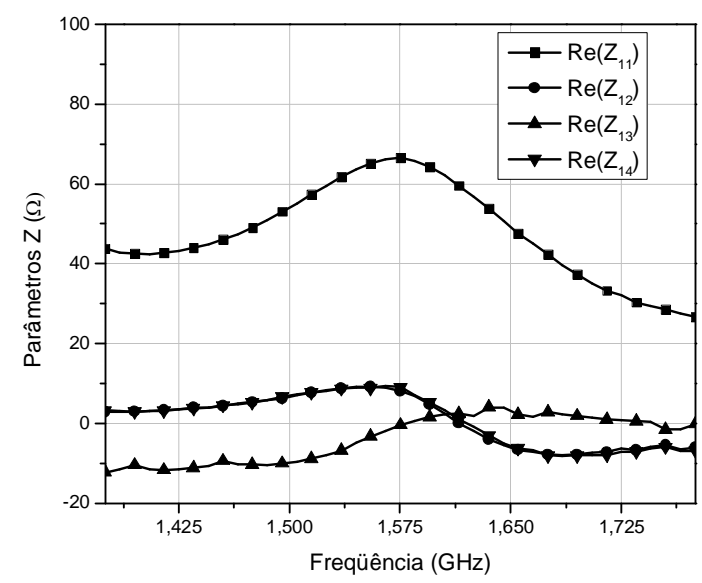

Fig. 6. Parâmetros impedância do elemento 1 da rede.

O diagrama de irradiação da rede, simulado como se medido fosse por um dipolo girante, é apresentado na Fig. 7 para o plano xz. Este tipo de diagrama possibilita avaliar o comportamento angular da razão axial da antena e, portanto, 
seu estado de polarização em determinada direção. Nota-se que a disposição dos elementos em rede propiciou, neste plano, a compensação da assimetria do diagrama do elemento isolado.

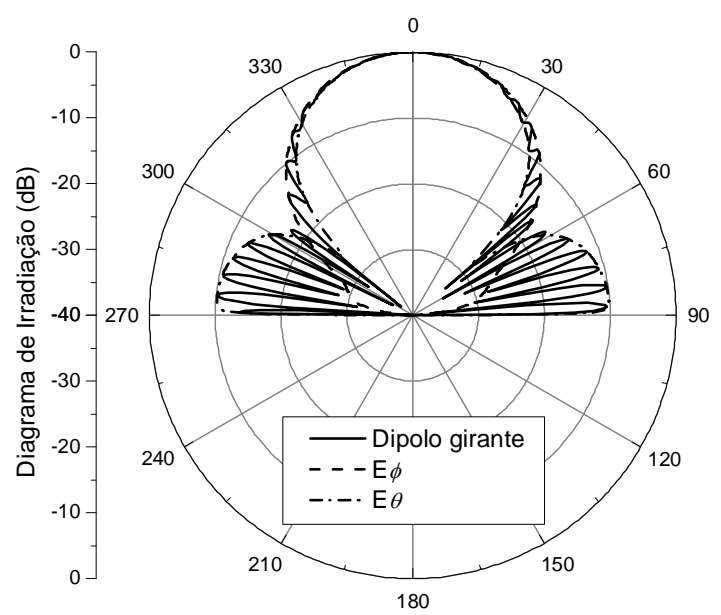

Fig. 7. Diagramas de irradiação da rede no plano xz, simulados na frequiência de $1,575 \mathrm{GHz}$

A Fig. 8 apresenta os gráficos do ganho $G_{0}$ e da razão axial na direção broadside. A razão axial apresenta valores abaixo de $0,5 \mathrm{~dB}$ ao longo da banda. De acordo com [5], este comportamento é esperado, uma vez que os elementos são alimentados por fontes de corrente isoladas. O sistema de alimentação a ser introduzido será o responsável pelo estreitamento da largura de banda da razão axial.

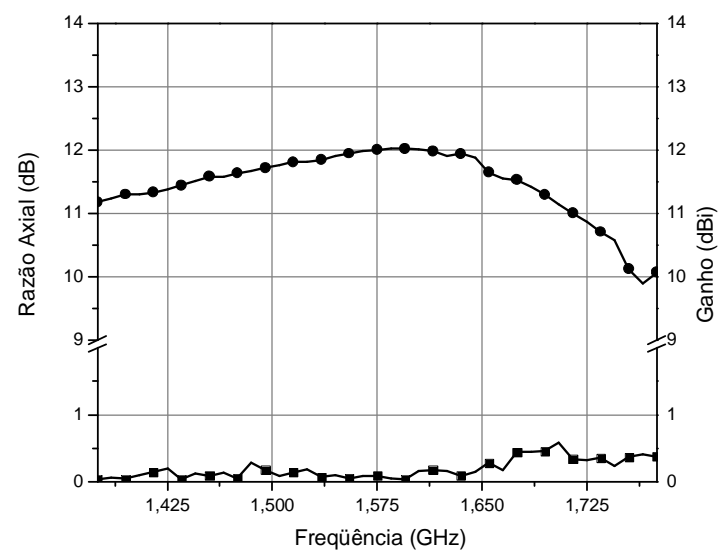

Fig. 8. Ganho $G_{0}$ e razão axial na direção broadside.

A impedância de entrada de cada elemento da rede é determinada a partir da equação abaixo

$Z_{i n N}=Z_{N 1} \frac{I_{1}}{I_{N}}+Z_{N 2} \frac{I_{2}}{I_{N}}+Z_{N 3} \frac{I_{3}}{I_{N}}+Z_{N 4} \frac{I_{4}}{I_{N}}$.

Na Tabela II estão apresentados os resultados obtidos para $Z_{\text {inN }}$. Observa-se que as impedâncias de entrada dos elementos da rede são equivalentes. Este resultado já era esperado, uma vez que as impedâncias mútuas entre as antenas $E$ são menores que as próprias.
TABELA II

IMPEDÂNCIA DE ENTRADA DOS ELEMENTOS DA REDE.

\begin{tabular}{|c|c|}
\hline$N$ & $Z_{i n N}(\Omega)$ \\
\hline 1 & $66,2-i 6,6$ \\
\hline 2 & $65,3-i 6,4$ \\
\hline 3 & $62,4-i 6,0$ \\
\hline 4 & $65,9-i 6,4$ \\
\hline
\end{tabular}

\section{Projeto do Sistema de AlimentaçÃo}

Nesta seção é realizado o projeto do circuito de alimentação da rede. Inicialmente, visando estabelecer uma relação de compromisso entre desempenho elétrico e dimensões físicas da antena, optou-se por projetar um circuito de alimentação constituído por divisores tipo $T$, linhas de transmissão e acoplador híbrido do tipo branch-line [9]. Neste projeto, as linhas que interconectam estes componentes são de $50 \Omega$.

Na Fig. 9 é mostrado o esquema elétrico proposto para este circuito. De acordo com este esquema, uma defasagem de $180^{\circ}$ é estabelecida entre as duas entradas dos divisores tipo $T$.

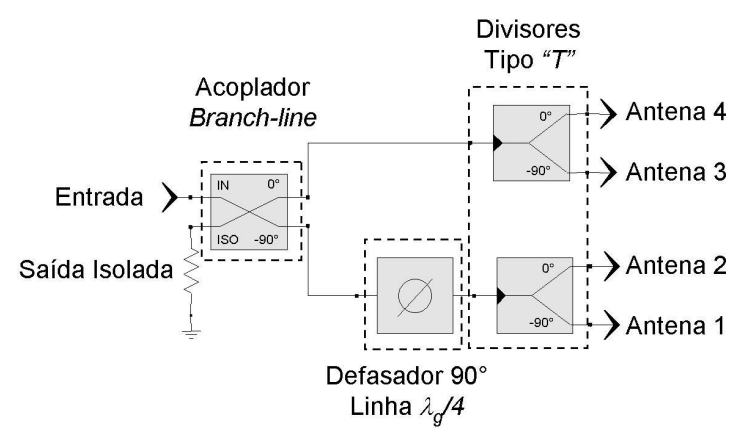

Fig. 9. Esquema elétrico do sistema de alimentação da rede.

Para a escolha do laminado a ser utilizado na construção do circuito de alimentação, três critérios foram adotados: limites físicos para a construção das linhas, comprimento de onda guiado médio e espessura máxima de substrato para supressão dos modos superiores ao $T M_{0}$ [10]. Para satisfazê-los escolheu-se o substrato $\mathrm{TMM}_{4}$ da Rogers Corporation, que possui $1,524 \mathrm{~mm}$ de espessura, $\varepsilon_{r}=4,5$ e 0,002 de tangente de perdas.

Para evitar irradiações espúrias na região frontal da rede, optou-se por implementar o circuito de alimentação na parte traseira do plano de terra. Em conseqüência, a conexão entre a antena $E$ e o circuito que a alimenta será feita através da transposição mostrada na Fig. 10. A Tabela III apresenta os resultados obtidos para as impedâncias de entrada vistas em planos de referência posicionados de forma a incorporar os efeitos das transposições. Comparando estes valores com os relacionados na Tabela II, nota-se que a transposição reduziu as partes reais das impedâncias de entrada e acentuou o seu caráter capacitivo. É importante salientar que neste estágio do projeto, as simulações ainda são realizadas sem que as cargas reais, isto é, as antenas $E$, estejam conectadas ao circuito.

Conhecidas as impedâncias de entrada (Tabela III) e as correntes de alimentação de cada elemento (Tabela I), parte-se para o projeto dos divisores tipo $T$. Este projeto é desenvolvido segundo a abordagem descrita em [11], a qual consiste em 
realizar uma seqüência de transformações de impedância partindo das impedâncias de entrada dos elementos - até o ponto da junção dos divisores, onde deverá ocorrer a mesma tensão para ambas as ramificações.

\section{TABELA III}

IMPEDÂNCIA DE ENTRADA DOS ELEMENTOS DA REDE COM TRANSPOSIÇÃOO PARA O CIRCUITO DE ALIMENTAÇÃO.

\begin{tabular}{|c|c|}
\hline$N$ & $Z_{i n N}(\Omega)$ \\
\hline 1 & $40,8-i 19,1$ \\
\hline 2 & $39,9-i 19,2$ \\
\hline 3 & $39,6-i 20,4$ \\
\hline 4 & $41,8-i 22,7$ \\
\hline
\end{tabular}

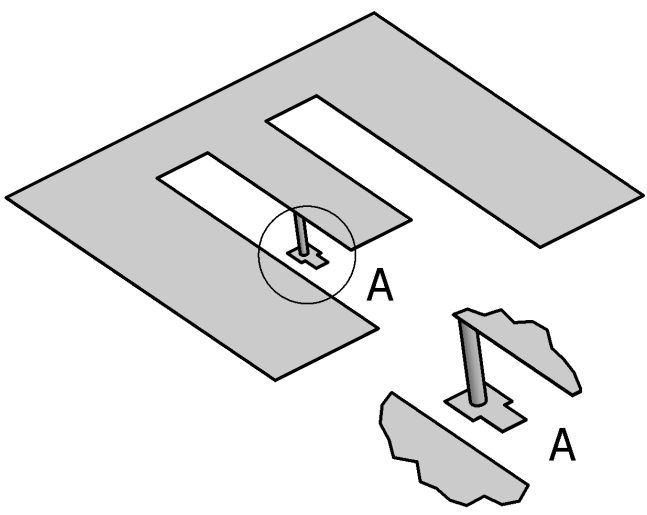

Fig. 10. Transposição da alimentação de uma das antenas da rede.

A Tabela IV relaciona as impedâncias e comprimentos elétricos resultantes para as linhas de microfita do sistema alimentador.

TABELA IV

IMPEDÂNCIAS CARACTERÍSTICAS E COMPRIMENTOS DAS LINHAS CONECTADAS ÀS CARGAS.

\begin{tabular}{|c|c|c|}
\hline Linha & $Z(\Omega)$ & $l\left(^{\circ}\right)$ \\
\hline 1 & 70,0 & 24,4 \\
\hline 2 & 68,0 & 90,0 \\
\hline 3 & 100,9 & 91,0 \\
\hline 4 & 70,0 & 26,2 \\
\hline 5 & 67,4 & 90,0 \\
\hline 6 & 70,0 & 23,2 \\
\hline 7 & 69,3 & 90,0 \\
\hline 8 & 101,6 & 92,7 \\
\hline 9 & 70,0 & 27,0 \\
\hline 10 & 68,2 & 90,0 \\
\hline 11 & 50,0 & 133,0 \\
\hline 12 & 50,0 & 43,0 \\
\hline
\end{tabular}

O pré-projeto do acoplador foi desenvolvido no ambiente do software GENESYS [12] com auxílio do modelo elétrico de linhas de transmissão em microfita. Já os modelos físicos dos divisores tipo $T$ e do acoplador híbrido foram simulados e otimizados no Ansoft Designer.

Com os divisores e o acoplador otimizados isoladamente, projetou-se o sistema alimentador mostrado na Fig. 11. Dois trechos de $50 \Omega$ de comprimentos adequados foram introduzidos para conectar as saídas do acoplador aos divisores tipo $T$ e proporcionar a necessária defasagem entre os elementos.

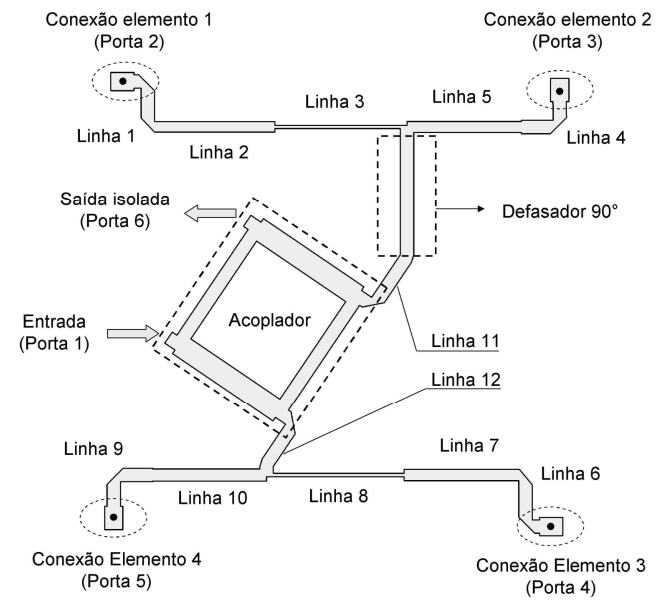

Fig. 11. Esquema final do circuito alimentador.

Observando a geometria apresentada na Fig. 11, nota-se que as linhas que conectam as portas 2 e 4 às junções possuem um trecho adicional (linhas 3 e 8). Este serve para ajustar a fase da tensão no ponto de conexão, uma vez que a amplitude já se encontra estabelecida após a transformação efetuada pelos trechos 2 e 7. Ressalta-se, ainda, que os comprimentos elétricos das linhas 3 e 8 são de aproximadamente $\lambda_{g} / 4$, muito próximo à defasagem relativa entre as correntes dos elementos tomados dois a dois, consecutivamente.

Projetado o sistema alimentador, seu desempenho foi avaliado através de simulação no Ansoft Designer. Para isto, os terminais de conexão dos elementos foram carregados com impedâncias concentradas dadas pela Tabela III e a porta 6 com uma carga casada (50 $\Omega$ ). Os resultados são apresentados nas Figs. 12 a 14.

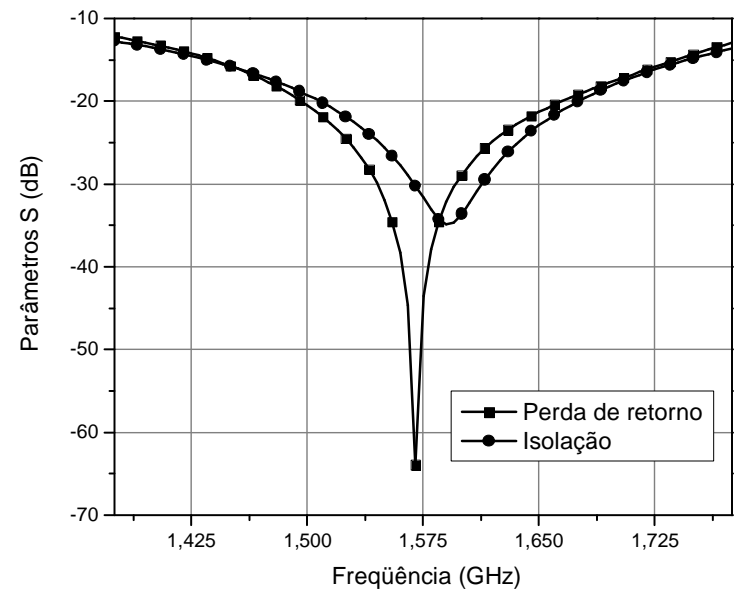

Fig. 12. Perda de retorno e isolação da híbrida carregada.

Na Fig. 12 são mostradas curvas para a perda de retorno e da isolação da híbrida carregada. Observa-se haver uma alta isolação na frequiência de projeto e uma perda de retorno melhor que $-40 \mathrm{~dB}$, nesta mesma freqüência.

A divisão de potência entre os terminais de conexão das antenas (Fig. 13) se encontra equilibrada na freqüência central. 
Afastando-se desta, como esperado, a divisão de potência tende a se desequilibrar devido ao emprego de componentes construídos com transformadores de um quarto de onda.

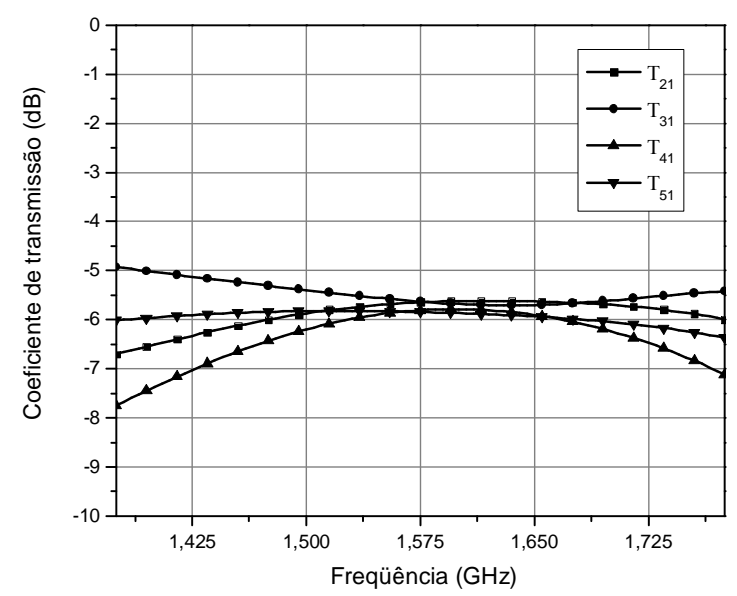

Fig. 13. Divisão de potência entre os terminais do divisor.

As defasagens estabelecidas entre os terminais de saída dos divisores são mostradas na Fig. 14.

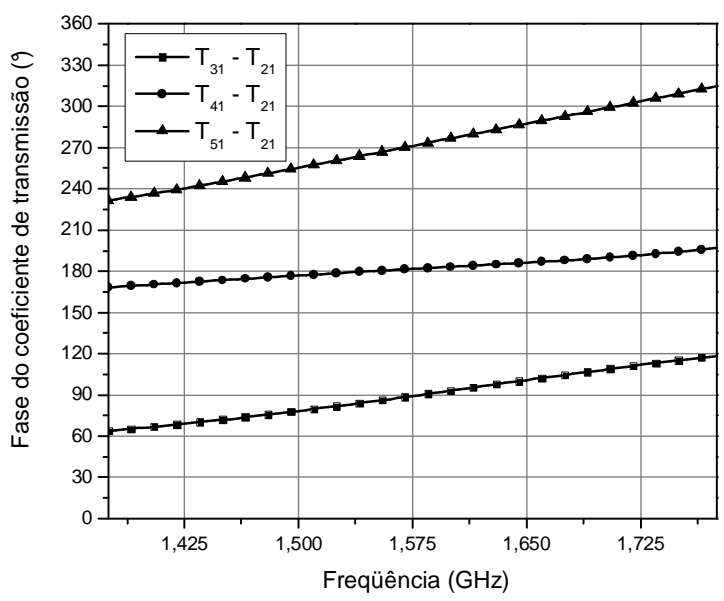

Fig. 14. Defasagem entre os terminais de saída dos divisores.

\section{Resultados Finais dA REDE $E$ CP}

A geometria completa da rede, incluindo o sistema alimentador (Fig. 15), foi simulada no ambiente do Ansoft Designer. Na Fig. 16 é apresentado o diagrama de irradiação no plano xz, simulado como se medido fosse por um dipolo girante, na freqüência de operação. Nota-se que o referido diagrama é equivalente ao apresentado na Fig. 7, permitindo concluir que o sistema de alimentação supriu adequadamente as antenas $E$ da rede, com as correntes descritas na Tabela I. Salienta-se ainda que o diagrama na parte posterior da rede é proveniente das correntes no sistema de alimentação.

O comportamento angular do referido diagrama mostra que a rede é capaz de gerar irradiação $\mathrm{CP}$ com baixa razão axial angular. Na Fig. 17 é apresentado o comportamento da razão axial ao longo de uma superfície cônica definida por $\theta=19^{\circ}$, a qual mantém-se inferior a $3 \mathrm{~dB}$ para qualquer posição angular $\phi$.

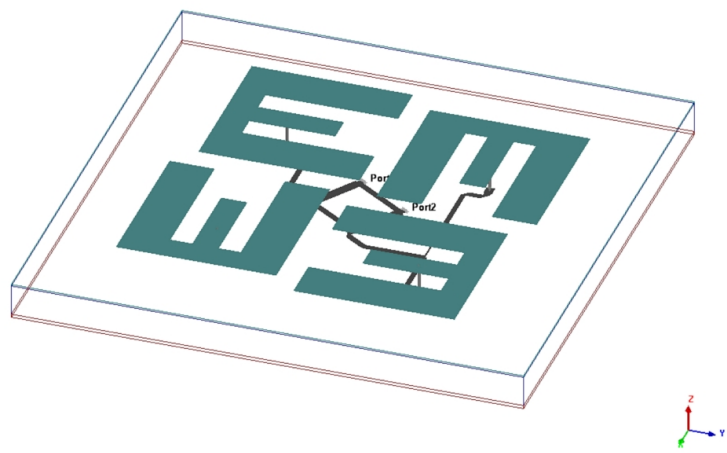

Fig. 15. Esquema completo da rede no ambiente do Ansoft Designer.

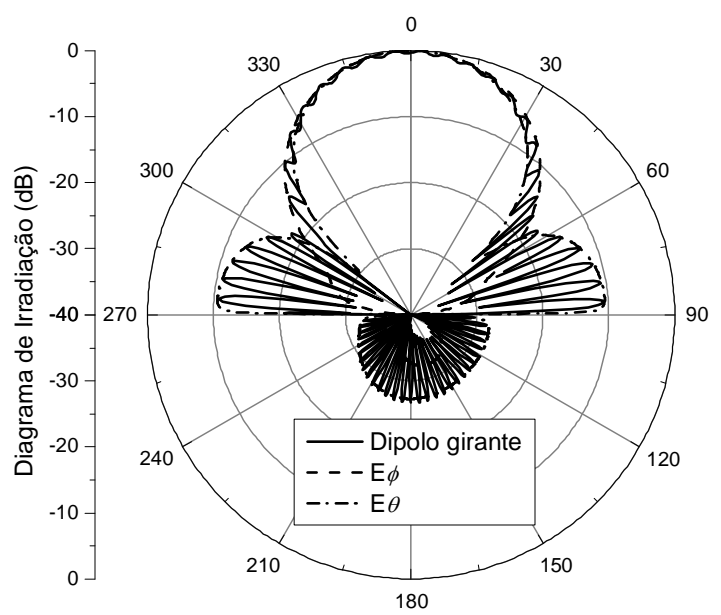

Fig. 16. Diagrama de irradiação da rede no plano xz, traçado com o sistema de alimentação incorporado.

Na direção broadside, a razão axial em função da frequiência apresentou o comportamento mostrado na Fig. 18, com uma faixa de passagem de $3 \mathrm{~dB}$ da ordem de $12,2 \%$, limite este imposto pelo sistema de alimentação. Nesta mesma figura encontra-se traçado o gráfico para o ganho $G_{0}$ da rede. Observase que o sistema de alimentação não modificou substancialmente este parâmetro.

Já as curvas obtidas para a isolação da híbrida e para a perda de retorno, na sua porta de entrada, são mostradas na Fig. 19. Nota-se que esse último parâmetro apresenta $14,1 \%$ de largura de banda de $-15 \mathrm{~dB}$, valor este maior do que a da razão axial acima mencionada. Tal comportamento se deve ao fato das fases das correntes de alimentação serem mais sensíveis às variações em freqüência dos componentes que constituem o sistema de alimentação.

\section{CONCLUSÕES}

A rede de antenas $E$, projetada para operar na condição $\mathrm{CP}$, através de alimentação uniforme e fase seqüencial, conseguiu prover irradiação CP com faixa de passagem de razão axial superior a $10 \%$. Tal fato se deve, principalmente, à escolha do elemento irradiador linearmente polarizado, que apresentou 
uma faixa de passagem de impedância, em rede, da ordem de $17 \%$.

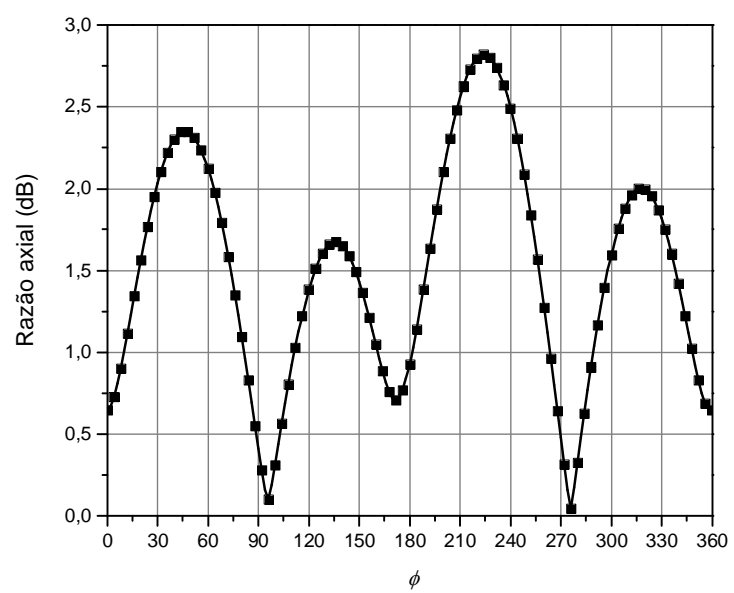

Fig. 17. Razão axial na freqüência de operação, calculada em $\theta=19^{\circ}$.

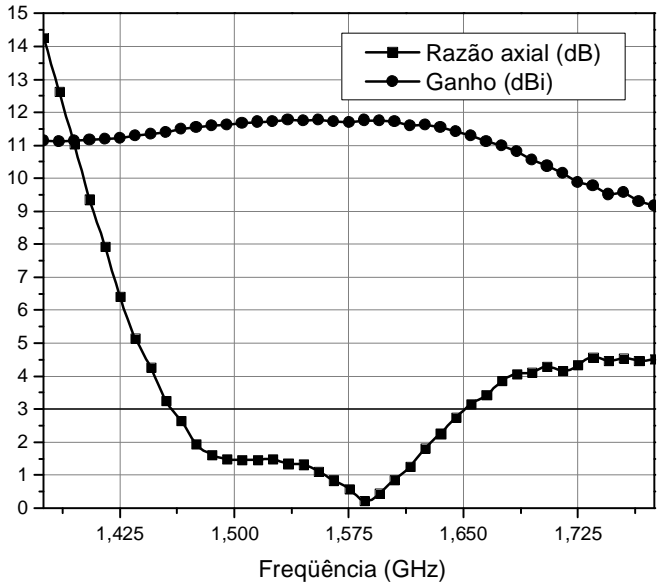

Fig. 18. Ganho $G_{0}$ e razão axial na direção broadside.

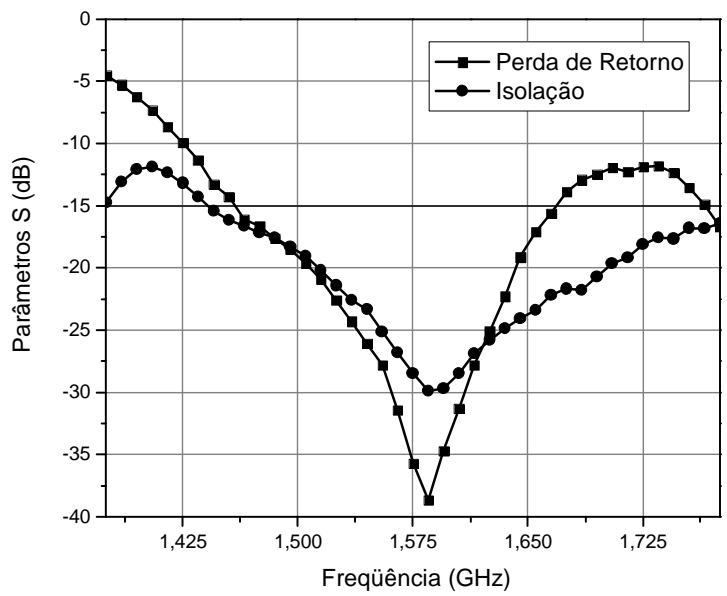

Fig. 19. Perda de retorno na porta de entrada da híbrida carregada e sua respectiva isolação.
A escolha do elemento, com faixa de passagem adequada, é primordial, visto que a razão axial é dependente do desempenho da impedância de entrada de cada um dos elementos da rede, bem como dos componentes utilizados para compor o circuito de alimentação da rede.

\section{REFERÊNCIAS}

[1] M. Thiel and A. Dreher, "Sequential rotation in a smart antenna terminal for broadband communication", IEEE Antennas and Propagation Society International Symposium, vol. 1, pp. 145-148, June 2004.

[2] J.R. James and P.S. Hall, Handbook of microstrip antenna, London: Peter Peregrinus, 1989.

[3] K.D. Palmer, J.H. Cloete and J.J. van Tonder, "Bandwidth improvement of circularly polarised arrays using sequential rotation", IEEE Antennas and Propagation Society International Symposium, Digest, vol. 1, pp. 135-138, July 1992.

[4] J. Huang, "A technique for an array to generate circular polarization with linearly polarized elements”, IEEE Trans. Antennas Propagat., vol. 34, pp. 1113-1124, Sept. 1986.

[5] S.S. Yang et al, "Study on sequential feeding networks for subarrays of circularly polarized elliptical dielectric resonator antenna", IEEE Trans. Antennas Propagat, vol. 55, pp. 321-333, Feb. 2007.

[6] F. Yang, et al., "Wide-band E-shaped patch antennas for wireless communication”, IEEE Trans. Antennas Propagat, vol. 49, pp. 1094 1100 , July 2001.

[7] D.C. Lunardi, "Rede de antenas de microfita circularmente polarizada com comutação de feixe e controle de nível de lóbulos secundários", Tese de Mestrado, ITA, 2007.

[8] Ansoft Designer, Ansoft Corporation. Disponível em: http://www.ansoft.com.

[9] D.M. Pozar, Microwave engeneering. 2. ed. New York: John Wiley, 1998

[10] T.Edwards, Foundation for microstrip circuits design. 2. ed. Chichester: John Wiley, 1992.

[11] D.C.M. Maciel, "Rede de antenas de microfita circularmente polarizada com apontamento de feixe e controle de nível de lóbulos secundários," Tese de Mestrado, ITA, 2005.

[12] GENESYS, Agilent Technologies. Disponível em http://eesof.tm.agilent.com/. 